\title{
Medienkompetenz als unbekannte Praxis. Ethnographische Perspektiven auf Digital Natives
}

\author{
Jochen Lange $\mathbb{D}$
}

Online publiziert: 13. Januar 2020

(C) Der/die Autor(en) 2020

Zusammenfassung Medienkompetenz ist vielfach normativ definiert und quantitativ geprüft. Eine andere Perspektive einnehmend, greift der vorliegende Beitrag die Debatten um Medienkompetenz von „Digital Natives“ auf, indem er die ethnologische Metapher kulturanalytisch fokussiert. Mit Bezügen zur neueren Kindheitsforschung geht es explorativ um die kulturspezifische Entdeckung von Medienkompetenz als noch unbekannte Praxis. Ethnographisch wird aufgezeigt, wie sich diese Medienkompetenz von Kindern in der Schule konstituiert, beschreiben und verstehen lässt. Dabei tritt die situative Verwicklung dieser Kompetenz mit Grundschule analytisch hervor.

Schlüsselwörter Medienkompetenz · Ethnographie · „Digital Natives“ · Schulund Kindheitskultur 


\title{
Media competence as an unknown practice. Ethnographic perspectives on digital natives
}

\begin{abstract}
Media competence is often normatively defined and quantitatively tested. This paper takes up the debates about the media competence of "digital natives", but looks for a different perspective. The ethnological metaphor is focused in a culturalanalytical way. With references to social childhood studies, the paper focuses on the culturally specific exploration of media competence as a still unknown practice. Ethnographically, it shows how the media competence of children is constituted at school, how it can be described and understood. The situational entanglement of this competence with primary school emerges analytically.
\end{abstract}

Keywords Media Competence · Ethnography · "Digital Natives" · Cultural Studies of School and Childhood

\section{Einleitung}

Die Digitalisierung ist zum Schlagwort unserer Zeit avanciert und wird mit vielfältigen Chancen sowie Herausforderungen für die Gesellschaft verknüpft. Zunehmend wird dabei ein Update der Mediendidaktik und Medienerziehung in Schulen gefordert und finanziell gefördert - prominent etwa mit dem „DigitalPakt Schule“. Insbesondere dem Begriff der Medienkompetenz wird dabei eine große Bedeutung zugesprochen, was sich bildungspolitisch z. B. mit dem Kompetenzrahmen „Bildung in der digitalen Welt" zeigt, den die Kultusministerkonferenz für die Schulen der Bundesländer verbindlich definierte (KMK 2016). Dieses definitorische Bestimmen von Medienkompetenz, die sich dann als Modell an Bildungsinstitutionen herantragen lässt, hat eine Tradition, der sich der vorliegende Beitrag in einem ersten Schritt annimmt. Medienkompetenz wird dabei als spezifisch theoretische und normative Figur rezipiert, der die Logik einer (kompetenz-)prüfenden Empirie folgt (Abschn. 2). Diese prominente Perspektive auf Kinder und Medienkompetenz wird (Abschn. 2.1) beispielhaft mit dem Diskurs und Forschungsstand zu „Digitale Natives“ veranschaulicht. Die vergleichsweise dichte Forschungslage zu der Frage, ob Kinder „Digital Natives“ oder „digitale Analphabeten“ (Leuthner 2014) seien, wird mit dem Beitrag um eine Perspektive erweitert, die sich der ethnologischen Metapher des Natives kulturanalytisch nähert. Dies geschieht, indem Kinder - unter Anschluss an die neuere Kindheitsforschung - als kompetente Akteure ihrer Lebenswelt betrachtet werden (Abschn. 2.2). Damit steht nicht die Frage im Zentrum, ob Kinder gemäß erwachsener Modellvorstellungen medienkompetent sind, vielmehr wird gefragt, wie Kinder medienkompetent sind. Eben diese Frage bearbeitet der Beitrag empirisch. Das ethnographisch-explorative Interesse (Abschn. 3) ist darauf gerichtet, wie sich die Medienkompetenz von Kindern in der Schule konstituiert, beschreiben und verstehen lässt. Der transparentmachenden Darlegung des forschungsmethodischen Vorgehens (Abschn. 3.1) folgt das empirische Material mit seinen Analysen (Abschn. 3.2 bis 3.4): Dichte Beschreibungen stellen den Kern des Beitrags dar und 
fokussieren letztlich (Abschn. 4) die praktische Hervorbringung von empirischer Medienkompetenz in ihrer schulkulturellen Verwicklung.

\section{Kompetenzorientierte Medienpädagogik}

Die Befähigung zum ,richtigen“ Medienumgang ist ein medienpädagogisches Leitmotiv und führte - unübersehbar ab den 90er Jahren - zum Medienkompetenzbegriff. Die Verwendung des Singulars („Medienkompetenzbegriff“) vermag darüber hinwegtäuschen, dass die Medienpädagogik einen erheblichen Aufwand in die elaborierende Ausdifferenzierung und etablierende Abgrenzung verschiedener Begriffsarchitekturen investierte, die das normative Soll der Mediennutzung mit Kompetenzkaskaden modellieren. Bereits 1996 titelte Kübler ausdrucksvoll mit „Kompetenz der Kompetenz der Kompetenz ... Anmerkungen zur Lieblingsmetapher der Medienpädagogik". Auch wenn sich die Akzentuierungen der Modelle unterscheiden, lieferten die Frankfurter Schule, Brechts Radiotheorie bzw. das kritisch-materialistische Medienkonzept bedeutende (medien-)theoretische Paradigmen und Bezugspunkte für die entwickelten Zielvorstellungen und Begriffe im deutschsprachigen Raum. Zudem ging eine gewisse Empirieskepsis mit der Grundsteinlegung der kompetenzorientierten Medienpädagogik einher: „Empirie und Erfahrungswissenschaft reproduzieren nur beschreibend und zählend, was war und ist. Diese Naivität als Verdinglichung wurde im Hinweis darauf, dass Erkenntnis und Interesse einander bestimmen, gesellschaftliche Zustände nicht unwandelbarer Natur sind, von der kritischen Medientheorie aufgedeckt" (Baacke 1974, S. 16). Vor diesem dominanten Theorie- und Normhintergrund entwickelte Baacke seine begriffsarchitektonische Pionierarbeit: Sein Medienkompetenzkonzept gliedert er in die zwei kategorischen Dimensionen der Vermittlung und der zielorientierten Handlung. Diese beiden Bereiche teilen sich erneut in je zwei Dimensionen: Vermittlung spaltet Baacke in Medienkritik und Medienkunde, Handlung wiederum in Mediennutzung und Mediengestaltung (vgl. Baacke 1999, S. 34). Medienkritik steht dabei - und bei Arbeiten die Baacke folgten - an prominenter Stelle. Nach Tulodziecki läuft Medienkompetenz z. B. auf die Fähigkeiten hinaus, (1) Medienangebote sinnvoll auszuwählen und zu nutzen, (2) eigene Medien zu gestalten und zu verbreiten, (3) Mediengestaltungen $\mathrm{zu}$ verstehen und zu bewerten, (4) Medieneinflüsse zu erkennen und aufzuarbeiten, (5) Bedingungen der Medienproduktion und -verbreitung analysierend zu erfassen, sowie Einfluss auf die Entwicklung der Medienlandschaft zu nehmen (vgl. Tulodziecki 1997, S. 142 ff.). Deutlich wird der präskriptive - teils curricular anmutende Charakter der Modelle. Die Teilfähigkeiten von Tulodziecki möchten z. B. direkt als schulische „Aufgabenbereiche“ verstanden werden, die ,die Grundlage für verschiedene Unterrichtsentwürfe“ und für ein Kompetenzmodell im Sinne von ,empirisch prüfbaren Bildungsstandards“ liefern (vgl. Tulodziecki et al. 2018, S. 41). Die auch bildungspolitische - Konjunktur von Medienkompetenz ist damit nicht nur auf die Omnipräsenz (digitaler) Medien in unserem heutigen Alltag zurückzuführen, sondern sie speist sich auch durch eine gewisse Passung (wenn auch nicht Deckungsgleichheit) zum Kompetenzverständnis heutiger outputorientierter Curricula. So formuliert die Kultusministerkonferenz in ihrem Strategiepapier sechs Kompe- 
tenzbereiche für das Digitale: 1. Suchen, Verarbeiten und Aufbewahren; 2. Kommunizieren und Kooperieren; 3. Produzieren und Präsentieren; 4. Schützen und sicher Agieren; 5. Problemlösen und Handeln; 6. Analysieren und Reflektieren (vgl. KMK 2016, S. 10-13). Unter diesen Bereichen finden sich Spezifizierungen wie z. B. „Als selbstbestimmter Bürger aktiv an der Gesellschaft teilhaben“ (KMK 2016, S. 11) oder auch ,Algorithmische Strukturen in genutzten digitalen Tools erkennen und formulieren" (KMK 2016, S. 13). Die Frage, woher diese Kompetenzen stammen, beantwortet die KMK dahingehend, dass für das Strategiepapier ,drei bekannte und bewährte Kompetenzmodelle herangezogen“"wurden (KMK 2016, S. 10). Diese einfließenden medienpädagogischen Modelle wurden teils übernommen, umgruppiert, modifiziert, gekürzt, umstrukturiert - kurzum: zu einer eigenen Mixtur verwertet (kritisch hierzu Tulodziecki et al. 2018, S. 48). Betrachtet man die für das „Mashup“ der KMK herangezogenen Modelle, so hat dieses (re-)kombinatorische Vorgehen eine gewisse Konsequenz: z. B. handelt es sich bei dem prominent zugrunde gelegten „DigComp“-Modell ebenfalls um eine aufwändig erstellte Kombination von konzeptionellen „Digital Competence“-Begriffen aus unterschiedlichsten Quellen. Die zugehörige Studie setzt sich im Kern aus einer umfangreichen Literaturschau zusammen, die den terminologischen Fachdiskurs unter Berücksichtigung vieler ,,review of concepts"-Papers resümiert (vgl. Ala-Mutka 2011), sowie einer Teilstudie, die sich der Implementierung von „Digital Competence“ nähert, indem primär dokumentenanalytisch Webseiten und Broschüren betrachtet werden (vgl. Ferrari 2012, S. 3). Durch ein stetiges Updaten und Erweitern (DigComp 2.0, 2.1 usw.) bringt es dieser Referenzrahmen inzwischen auf 21 Kompetenzen, die sich unter fünf Bereiche subsummieren (1. Information and data literacy; 2. Communication and collaboration; 3. Digital content creation; 4. Safety; 5. Problem solving), zu denen überdies noch acht Kompetenzstufen (,,proficiency levels“") gehören (vgl. Carretero et al. 2017, S. 10-18). Vor diesem skizzierten Hintergrund kann bei Leserinnen und Lesern zuweilen der Eindruck entstehen, dass sich ein Schneeballeffekt beobachten lässt: Ein Kompetenzkarussell hat Fahrt aufgenommen und generiert einen beträchtlichen Teil seines Schwungs referenziell aus sich selbst heraus. Die diskursiv aufeinander verweisenden Begriffsmodellierungen produzieren dabei einen gewissen Normüberschuss. Der Prozess der Theorie- und Begriffsbildung könnte durch heterogene Empirie bereichert werden. Obgleich in der Vergangenheit viele empirische Arbeiten erschienen sind, ist ,nicht zu übersehen, dass der Stand der medienpädagogischen Forschung in mancher Hinsicht ungenügend geblieben ist. So ist die Zahl medienpädagogischer Forschungsarbeiten für eine expansive Wissenschaftsdisziplin letztlich zu gering geblieben“ (Moser 2014, S. 56). Eine gewisse Tradition haben zum einen Befragungsstudien, die (Selbst-)Aussagen zur Mediennutzung quantifizieren und einen eher informierenden als theoriebildenden Charakter haben (vgl. z. B. Medienpädagogischer Forschungsverbund Südwest 2019), zum anderen finden sich relativ viele Forschungsarbeiten, die die vorab festgeschriebenen - also theoretisch „mitgebrachten“ - Medienkompetenzbegriffe empirisch zu füttern, wiederzufinden bzw. diagnostizierend zu evaluieren pflegen (vgl. z. B. Bos et al. 2014; Treumann et al. 2007). Trotz teils qualitativer bzw. triangulierender Forschungsanteile ist dabei insgesamt eine quantitative Prüflogik leitend, die sich aus der theoretischen Ver- 
wurzelung begründen lässt: „Theorien der (Medien-)Kompetenz sind, mit anderen Worten, zu sehr auf statische Lenresultate fokussiert“" (Sutter 2014, S. 73).

Vor dem Hintergrund dieser normativen, theoretischen und empirischen Ausprägungen hat sich auch der Diskurs um „Digital Natives“ konstituiert, den ich im Folgenden als Beispiel für die dominierende Perspektive auf Kinder und Medienkompetenz vorstellen werde. Angeschlossen nutze ich ihn für die kontrastierende Schärfung meines Forschungsvorhabens, das sich ergänzend um eine andere Perspektivierung bemüht.

\subsection{Atteste der Medienkompetenz}

Die Gegenüberstellung der Metaphern „Digital Native“ und „Digital Immigrants“ wurde von Prensky in den (medien-)pädagogischen Diskurs eingespeist. Er postuliert, dass heutige Schülerinnen und Schüler (,Digitale Natives“) anders sind als die Erwachsenen („Digital Immigrants“). „Digitale Natives“ würden anders lernen, denken und arbeiten (Fähigkeit des Multitaskings, Effizienz, Präferenz zur Vernetzungen, Durchblick in der digitalen Welt usw.). An diese Kompetenzen schließt Prensky sein Plädoyer für eine Reform von Bildungsinstitutionen mit ihren tradierten Vermittlungsprozessen an (vgl. Prensky 2001, S. 1-4). Vor dem skizzierten Hintergrund einer kompetenzprüfenden und nutzungsquantifizierenden Medienpädagogik provozierten die unterstellten Kompetenzen selbstverständlich Reaktionen: „Are ,digital natives“ really digitally competent?“ (Li und Ranieri 2010). Zeitnah wurde eine Dekonstruktion der „Mystifizierung“ postuliert (vgl. z. B. Schulmeister 2012). Die Frage, ob Kinder „Digital Natives“ oder „Digitale Analphabeten“ seien (vgl. Leuthner 2014), füllt umfassende Arbeiten und wird häufig in folgender Weise beantwortet: „Kinder haben vielfältige Probleme im Umgang mit Computer und Internet. [...] So verfügen viele Kinder, auch etliche der schon älteren, nur über dürftige technische Basiskompetenzen“ (Leuthner 2014, S. 271). Erst recht sei Kindern „,keine ausreichende Informationskompetenz“ zuzusprechen, ihre „Unbefangenheit, die bloße Verfügbarkeit der Technik und deren Nutzung an sich, darf nicht mit Medienkompetenz verwechselt werden“ (Leuthner 2014, S. 273).

Fraglos können Prenskys Postulate, was die „Digital Natives“ angeblich auszeichnet, leicht problematisiert werden. Dennoch hat er mit den Begriffen der „Digital Natives“ und „Digital Immigrants“ äußerst spannende Bilder eingebracht, deren Erkenntnispotenziale man nicht allein mit der oben skizzierten Empirie ausschöpfen kann. Bisher wurde die Denkfigur der „Natives“ und „Immigrants“ vorwiegend aufgenommen, um aus der eigenen (Erwachsenen-)Kultur heraus ein Kompetenzraster zu entwickeln und dieses an die jungen Natives anzulegen. Die ethnologischen Metaphern können aber auch dazu anregen, die kulturelle Spezifik von Medienkompetenzen pädagogisch zu reflektieren. Was Medienkompetenz ist, lässt sich schwerlich kulturunabhängig oder -übergreifend bestimmen. Dies zeigt sich nicht nur anekdotisch an dem Beispiel, dass sich US-amerikanische Journalisten wie Jeff Jarvis irritiert-befremdlich darüber äußern, dass es in Deutschland Menschen gibt, die ihre Hausfassade in Google Street View verpixeln lassen wollen, aber keine Probleme damit haben, nackt eine öffentliche Sauna zu betreten. Auch bei dem oben zitierten DigComp-Referenzrahmen - der einen internationalen Diskurs rezipiert, dabei aber 
europäisch akzentuiert bleibt - ist auffällig, dass das Kernanliegen der deutschsprachigen Medienpädagogik (die im Zentrum stehende gesellschaftsbezogene kritische Reflexion) deutlich weniger prominent und explizit vorkommt (vgl. z. B. Carretero et al. 2017, S. 21). Mit derartigen Differenzen - die hier beispielhaft lediglich innerhalb der westlichen Welt aufgezeigt sind - relativiert sich Medienkompetenz und zerfällt in kulturspezifische Normen sowie Aktivitäten. Damit soll die für Kinder geforderte Enkulturationshilfe beim Medienumgang (vgl. z. B. Kammerl 2014, S. 21) nicht in ihrer pädagogischen Legitimation beschnitten werden. Für die Forschung geht es mir mit der kulturellen Relativierung von Medienkompetenz um eine Steigerung der Reflexivität, die sich über die kulturelle „Fremdheit“ zwischen Kindern und Erwachsenen begründet und zu neuen Verständnisweisen führen soll (vgl. Honig et al. 1999, S. 9).

\subsection{Explorationen der Medienkompetenz}

In der Tradition, die „Welt des Kindes“ besser zu verstehen (Muchow und Muchow 1935, S. 69), geht es der neueren, sozialwissenschaftlichen Kindheitsforschung um die Analyse alltäglicher Interaktionen und Prozesse (vgl. z. B. Krappmann und Oswald 1995; Zinnecker 2001). Damit verschiebt sich der Blick auf Kindheit, weg von ihrer Betrachtung als unabgeschlossen Entwicklungsphase, hin zur „Eigenständigkeit von Kinderkultur" (Kelle und Breidenstein 1996, S. 54), die sich durch Praktiken in situ konstituiert. Der Forschung geht es um die „Beschreibung der Herstellung sozialer Situationen und sinnhafter Ordnungen durch Kinder" (Heinzel 2003, S. 128). Analytisch zeigen sich Kinder dabei als kompetente Akteure, die sich mit Kreativität, Vitalität und Energie ,eine bedeutungsvolle und lebenswerte Welt schaffen“ (Hengst 2008, S. 564). Begreift man Kindheiten in diesem Sinne als eigene Kulturen, so ist die Frage nicht ob, sondern wie Kinder medienkompetent sind. Empirisch (und nicht normativ) erscheint es wenig plausibel, warum erwachsene und kindliche Medienkompetenzen einander gleichen sollten. Versteht man Medienkompetenz nicht als gesetzte Norm, lässt sich ein Explorationsanliegen forcieren, mit dem kindliche Medienkompetenz als eine noch weitgehend unbekannte Figuration gesehen wird, die sich im alltäglichen Miteinander konstituiert. Nach Facetten und Verständnisweisen von Medienkompetenz suche ich somit im Feld. Die Metapher des „Digital Natives“ möchte ich damit empirisch zu ihrer kulturanalytischen Verortung zurückführen, die eine Erforschung in ethnologischer Linie offeriert. Vor diesem Hintergrund teile ich mit der neueren Kindheitsforschung ihr methodisches „Plädoyer für Ethnografie“ (Hengst 2008, S. 562f.). Praxeologische Arbeiten, die ein ethnographisches Interesse am Alltag mit Fragen nach neuen Medien verbinden, sind rar. „Praxisforschung stellt eine Lücke in der methodologischen Diskussion der Medienpädagogik dar, die zunehmend erkannt wird" (Moser 2014, S. 55; Herv. i. Orig.). Auffällig ist dabei, dass verschiedene Beiträge praxeologisch orientierte Erkenntnisinteressen formulieren, diese aber von ethnographischen Vorgehensweisen zu lösen versuchen. Dies reicht über ein Plädoyer für konversationsanalytische Forschungen (vgl. Sutter 2014, S. 87) ebenso hinaus, wie über das Formulieren des ,Anspruch[s] einer nicht-teilnehmenden Beobachtung“ (Münte-Goussar und Grünberger 2019, S. 928): Teils wird eine interviewbasierte Praxisforschung postuliert (vgl. Bettinger 2018, S. 591). Trotz 
eines tradiert-medienpädagogischen Interesses an der aktiven Medienaneignung unter besonderer Berücksichtigung der Lebenskontexte von Kindern (vgl. Schorb und Theunert 2000, S. 35), begeben sich Forscherinnen und Forscher erstaunlich selten zu den Orten, an denen derlei Aktivität gemacht und somit empirisch zugänglich wird.

\section{Medienkompetenz empirisch wenden}

\subsection{Erkenntnisinteressen und methodisches Vorgehen}

Vor dem Hintergrund der aufgezeigten Diskurse und Desiderate soll mit dem vorliegenden Artikel ein Beitrag zu einer praxeologischen Medienforschung geleistet werden, die an kulturanalytische Kindheits- bzw. Schulforschung anschließt. Zentral ist die Frage nach der Medienkompetenz von Kindern, ohne dass dabei theorie- bzw. kompetenzprüfend normative Modellvorstellungen von Medienkompetenz an Kinder herangetragen werden sollen. Vielmehr wird grundsätzlich von Kindern als kompetente Akteure ihrer mediendurchdrungenen Lebenswelt ausgegangen und explorativ gefragt, wie sich ihre (Medien-)Kompetenz konstituiert, beschreiben und verstehen lässt. Mit diesem Verweis auf kompetente Akteure ist nicht gemeint, dass Kinder stets problemlos mit den Medien agieren. Vielmehr wird angenommen, dass Kinder die Medien ihrer Lebenswelt samt sich ergebener Problemstellungen in konstruktive Prozesse der Sinnstiftung einbeziehen und sich dabei kulturspezifische Interaktionen, Befragungen, Deutungen, Einordnungen und implizite Strategien der Aneignung beobachten lassen:

Im Vergleich zu anderen qualitativen Forschungsstrategien, die zugleich auf Retrospektionen angelegt sind - wie bspw. Interviews - ermöglicht sie [die Ethnographie], schrittweise den performativen Vollzug der kindlichen Medienaneignung in den Blick zu nehmen und damit auch die Prozessualität von gegenwärtiger Sinnhaftigkeit und Bedeutung der Situationen zu rekonstruieren.

(Schulz 2014, S. 231)

Eben diesem Erkenntnisinteresse gehe ich mit der Beobachtung von schulischen Situationen nach. Grundsätzlich hat sich die Grundschule als ein erkenntnisproduktives Feld der neueren Kindheitsforschung erwiesen. Für mein spezifisches Forschungsinteresse, Medienkompetenzen in kulturellen Relationen und Kontrasten analytisch sichtbar zu machen, offeriert die Schule zudem ein besonders Erkenntnispotenzial, da hier Peer-, Schul- und Erwachsenenkultur zusammenlaufen. Die Relevanz des Feldes ist auch dadurch zu begründen, dass Schule zunehmend unter Druck gerät, die digitale Kluft zwischen medialem Vor- und Nachmittag zu schließen. Digitale Medien halten verstärkt und gefördert Einzug in die Schule, obwohl das empirische Wissen zum Wie ihrer alltäglichen Einbeziehung noch ausgesprochen dünn ist.

Das folgende empirische Material stammt aus der laufenden Forschung, in deren Rahmen bisher vier Klassenverbände, die von Schülerinnen und Schülern dritter sowie vierter Jahrgangsstufen besucht wurden, wiederkehrend von mir teilnehmend 
beobachtet wurden. Über bisher etwa neun Monate hinweg fokussierte ich die Interaktionen mit Schulcomputern, Tablets, Interaktiven Whiteboards u. a. Methodologisch zentral ist dabei die reflexive Präsenz im Feld, aus der über Feldnotizen (vgl. Emerson et al. 1995) letztlich ,dichte Beschreibungen“(Geertz 1983) von Alltag hervorgehen, die Erfahrungen mobilisieren (vgl. Amann und Hirschauer 1997, S. 30). Das Beschreiben der Beobachtungen ist somit ein erster analytischer Schritt der Sinnzuschreibung, bei dem nicht zwischen einer ,sachlichen Beschreibung“ und einer ,subjektiven Wahrnehmung“ getrennt werden kann oder soll: „Jede ,Beobachtung' von Verhalten [...] ist vor aller Interpretation bereits in der Wahrnehmung des Verhaltens durch den Beobachter geprägt“ (Beck und Scholz 2000, S. 150). Während die Feldnotizen in der Schule handschriftlich entstanden, ging mit der Weiterverarbeitung zu Beschreibungen ihre Digitalisierung einher. Zugleich wurden sie in die Analysesoftware „MAXQDA“ übertragen. Im Feld gemachte Fotos dienten dabei ebenso der textlichen Verdichtung wie digitale Bildschirmvideos, die teils das Geschehen auf den Monitoren mit einer Screen Capture Software einfingen. Die Beschreibungen wurden mit Bezug zur Grounded Theory weiter analytisch systematisiert. Neben dem grundsätzlichen Postulat einer theoriegenerierenden Empirie (vgl. Strauss 1994, S. 70), war das Zirkulieren von Schreibtisch- und Feldphasen ein bedeutendes Element für die Arbeit. Bei der Suche nach Gegenüberstellungen, Varianten und Kontrasten hat das einhergehende ,theoretical sampling“ eine große Relevanz für das (Wieder-)Entdecken und ausdifferenzierende Anpassen von Kategorien. Das so gesponnene kategoriale Netz beantwortet die Frage, worum es den Akteuren eigentlich in den Daten geht. Eben diese Frage ist es letztlich, auf die die Grounded Theory zielt (vgl. Strauss 1994, S. 66), und die von mir im Sinn des Geertz'schen ,what the hell is going on here?" verstanden wird. Die folgende Beschreibung einer beobachteten Situation führt als „Opener“ in das Feld und die Analysen ein.

\subsection{Erster Schultag für das Tablet}

Bei vielen Kindern im Klassenraum herrscht eine euphorische Spannung. Sie wissen was in der nun folgenden Stunde geplant ist, einige haben die Tablets - die in unscheinbarer brauner Pappe auf einem der vorderen Tische liegen - bereits erspäht, andere erinnern sich an das „,Versprechen“ der Lehrerin vom letzten Freitag. Die Lehrerin (Frau Berg) beginnt nun die Stunde und sagt, dass es an dem heutigen Montag ausnahmsweise keinen Erzählkreis zum vergangenen Wochenende geben wird. Ein Mädchen ruft enttäuscht „Oh, ne ... “ während viele andere Jungen und Mädchen ihr korrigierend ins Wort fallen: „Ey doch! iPads! “. Die Rufe scheinen das unzufriedene Mädchen daran erinnern zu wollen, was heute geplant ist. Die Lehrerin stimmt vielversprechend mit ein: „Der große Tag ... “. Daraufhin wird abgefragt, wer von den Kindern denn schon Erfahrungen mit Tablets hat. Viele Finger schießen in die Höhe und recken sich zur Decke. Anstelle des Erzählkreises zum Wochenende dürfen die Kinder nun von ihren Tablet-Erfahrungen berichten. Frau Berg hat währenddessen ein Tablet exemplarisch (und gut sichtbar) in der Hand, sie nutzt es als eine Art Vorführgerät zur unterstreichenden Illustration ausgewählter Aspekte der Kindererzählungen. Die Schülerinnen und Schüler berichten bunt gemischt darüber, wo und 
mit welchen Personen die Geräte zu Hause benutzt wurden, welche konkreten Spiele gespielt wurden und was man generell alles mit solchen Geräten machen kann. Nach einer Weile erklärt Frau Berg, was hier und heute gemacht werden soll: Es soll ein Text abgetippt werden. Frau Berg begibt sich zu dem Klassensatz der Geräte und erinnert noch einmal eindringlich an eine ihr wichtige Umgangsregel: „Nicht damit rumlaufen! ". Dann nimmt sie sich den Pappkarton mit den Tablets und teilt diese aus. Eines nach dem anderen wird den Schülerinnen und Schülern durch die Lehrerin vorgelegt. Ich muss unweigerlich an das Austeilen von Klassenarbeiten bzw. Schulheften denken. Pro Kind findet sich ein Gerät, das auf dem Tisch liegt und durch ein „Smart Cover“ verdeckt ist. Dieses Verdeck lässt sich umschlagen, ähnlich wie man ein Heft oder Buch aufschlägt - allerdings tut dies in der Situation noch niemand. Erst als fast alle Geräte ausgeteilt sind, kommen erste Frage: „Dürfen wir schon aufmachen?" Nachdem alle Kinder ihr Gerät haben und die Lehrerin den Beginn signalisiert, erfolgt das Aufschlagen fast synchron. Die Schülerinnen und Schüler suchen das Programm, einige finden es schnell, andere gar nicht, die Lehrerin und ich werden ab und zu zur Hilfe gerufen. Nach einer Weile haben alle ein leeres Dokument samt Tastatur auf dem Bildschirm und beginnen mit der Aufgabe (handschriftlichen Text eintippen). Eifrig wird geschrieben, getuschelt und gefragt. Ein Mädchen steht mit dem Gerät in der Hand auf und ein anderes Mädchen weist sie umgehend zurecht: „Kim, wir dürfen nicht [laufen] ... “ und die Lehrerin bestätigt, dass sie nicht möchte, dass ,,ihr mit den Geräten spazieren geht“. Kim setzt sich wieder bedächtig mit dem Gerät auf ihren Platz.

Auf den ersten Blick zeigt die Beobachtung eine Premiere: In der aufgesuchten Schule gelangen neu angeschaffte digitale Medien erstmals in den Unterrichtsalltag. Allerdings harmoniert die Beobachtung schwerlich mit dem vielfach zu lesendem Postulat, dass von den neuen Medien tiefgreifende Veränderungen der tradierten schulischen Praxis zu erwarten seien bzw. dass digitale Medien ein ,rethinking of existing conceptual frameworks and pedagogic practices“ verlangen (Buckingham 2007, S. 117). Vielmehr zeigt sich, wie die Geräte als schulische Medien situativ entstehen. Damit folge ich einem performativen Medienbegriff der betont, dass Dinge, Infrastrukturen usw. eben erst durch Gebrauchsweisen zu Medien werden (vgl. z. B. Münker 2013). Im Zuge der Einschulung werden Eigenschaften der neuen Medien, die in der außerschulischen Welt zentral und charakterstiftend sind, in schulischen Umgangsweisen aufgelöst: Der Tablet-Computer ist nicht länger ein „,mobile devices“, er erhält vielmehr den Status einer stationären Klassenarbeit und wird - in einer tradierten schulischen Ordnung - zu einem Schreibheft. Tablets in der Schule können sich damit recht grundsätzlich von Tablets in der Peer-Group oder Familie unterscheiden. Kinder sind als Schüler(innen) an der Hervorbringung dieser Andersartigkeit beteiligt, sie machen sie mit - und sie machen sie richtig mit, damit Schule und Medium zusammen funktionieren. Diese Kompatibilität wird kompetent hergestellt:

Kompetenter Medienumgang bedeutet, einen in der prinzipiell ungewissen Situation und im Zusammenspiel mit den anderen Partizipanden sowie unter Berücksichtigung des situativen Kontextes anerkennbaren Umgang zu finden. Dies erfordert ein flexibles und sensibles Vorgehen, wird durch Hinweise, Vor- 
und Nachmachen, Nichtbeachtung, Anerkennung etc. kollektiv und dynamisch vermittelt und ausgehandelt. (Rode und Stern 2017, S. 241; Herv. i. Orig.)

Medien und Medienkompetenz sind schulisch konstituiert und Kinder nähern sich ihnen in der Schule kontextsensibel. Dieser Spur wird im Folgenden weiter nachgegangen, indem die dominante schulische Kategorie des (Ab)Schreibens weiter fokussiert wird.

\subsection{Bedeutung aus der schulischen Ordnung}

Rechtschreibung: digitale Fehler Die Schüler(innen) können sich derzeit noch frei mit den Geräten beschäftigen. Mareike möchte die Internetseiten ,von Bibi und Tina“ besuchen. „Gut, kennst du die Adresse?" frage ich. Etwas zögerlicher antwortet Mareike: „Ja ... www Bibi und Tina ... Die muss ich dahin schreiben, ne?", fragt sie und zeigt auf das Eingabefeld der Adressleiste im Browser. „Genau“, antworte ich zustimmend. Mareike klickt in das Eingabefeld, beginnt zu tippen (,www, Bibi und tina“) und bestätigt erwartungsvoll. Das Resultat wirkt enttäuschend, der alte Browser gibt nur die Fehlermeldung aus, dass die URL falsch sei. Ich setze an: „Wir müssen uns noch mal die Adresse anschauen, da stimmt was nicht ... “. Die kleine Pause, die ich einlege, nutzt Janosch: „Klar stimmt da was nicht! " Er deutet auf die Adresse und korrigiert Mareike vorwurfsvoll: „,Tina“ schreibt man groß! Ist ein Name“. Mareike löscht das „t“ und ersetzt es durch ein „T“, bestätigt die Eingabe ein zweites Mal - aber der Erfolg bleibt erneut aus. Nach kurzem Überlegen und einem Hinweis von mir, wird (auf Janoschs Bildschirm) eine Suchmaschine mit der Zeichenkette ,Bibi und Tina' gefüttert. Bereits der Titel des ersten Suchergebnisses verrät den Erfolg: „Die offiziellen Seiten von Bibi und Tina - Abenteuer auf Schloß Falkenstein“. Ein paar Zeilen tiefer steht die (technisch) korrekte Schreibweise der Adresse („www.bibiundtina.de/“) und sorgt für zwei zweifelnde Gesichter. „Schreibt man also alles klein.“ sagt Mareike nachdenklich, Janosch entgegnet: „Ist aber falsch so ... “, „Das ist doch auch eigentlich nicht alles ein Wort“, moniert Mareike empört. „Nein“ bekräftigt Janosch. Beide werfen einen vergleichenden Blick auf Mareikes Bildschirm und das dort stehende „www, Bibi und Tina“. „Aber ein Komma gehört da wirklich nicht hin“, korrigiert Janosch und setzt nach: „Dass da ein Punkt hin muss weiß doch jeder: ,wwwPUNKT!"“.

In der Situation geht es um das technische Bedienen von Software, über richtige Texteingaben. Die Kinder wissen, dass - insbesondere in der Schule - auf eine korrekte Schreibweise zu achten ist, die sich aus Regeln speist (z.B. die Großschreibung von Namen). Um eben diese schulische Schreibweise sind sie bemüht und reagieren erst irritiert, dann pikiert darauf, dass ihnen der Schulcomputer Fehler unterstellt, obwohl es seine vermeintlich richtige Schreibweise ist, die voller Fehler sei. Die Orthographie des www ist eine andere als die Orthographie des Deutschunterrichts. Diese unerwartete Inkompatibilität, über die in der Situation sichtlich sinniert wird, hat für die Schüler(innen) etwas von einem ethnomethodologischen Krisenexperiment: ,Wir haben alles richtiggemacht, der Interaktionspartner verhält sich unangemessen“. Aus dem Scheitern des Versuchs, das Schulische und das Di- 
gitale zusammenlaufen zu lassen, führt abschließend jedoch erneut ein schulischer Weg als Notausgang: Das Auswendiglernen von kaum ableitbaren kultur-technischen Konventionen: Dass nach dem www ein Punkt folgt, muss man genauso als Information wissen, wie die Großschreibung von Substantiven.

Abgucken: digitaler Sichtschutz Johannes sitzt heute das erste Mal an dem Schulcomputer. Dieser hat den Startvorgang soeben abgeschlossen, das Betriebssystem verlangt nach einer Benutzeranmeldung. Der Junge blickt auf den ihm anscheinend unbekannten Anmeldedialog, der ihn vom Desktop mit seinen Programmen und Möglichkeiten trennt. Das Anmeldefenster hat zwei Eingabezeilen: Zum einen die Benutzerzeile (in dieser steht bereits der Eintrag „Schueler“), zum anderen die Eingabezeile für das "Passwort" (dieses Feld ist noch leer). Johannes wirkt kurz irritiert, fragt mich dann aber: „Wie ist das Passwort? “. Ich antworte „, das Passwort ist ,Kassel “. Der Junge macht sich daran, das Kennwort einzutippen. Dies führt zur nächsten Irritation: Anstelle der erwarteten Buchstaben erscheinen nur große Punkte in dem Eingabefeld. Er betrachtet kurz diese Punkte, guckt dann zu mir und murmelt: „Damit keiner abschreibt, ne?“.

Der Schüler scheint nur eine Sekunde irritiert zu denken, dass mit der Koppelung von Ein- und Ausgabe etwas nicht stimmt. Die Erklärung, warum der Schulcomputer die eingegebenen Buchstaben des Passworts nicht richtig darstellt, sondern in Punkte umwandelt, formuliert der Junge in schulischem Vokabular: Es soll am Abschreiben respektive Abgucken hindern. War das Schulische beim Korrigieren der Internetadresse noch primär Quelle einer Vorgehensstrategie, wird es nun zur Erklärungsgrundlage. Es wird zur Deutungsfolie, die an die digitale Sicherheitsmaßnahme angelegt wird und zu einer tragfähigen Sinnzuschreibung führt. Auch in diesem Sinne werden Bedeutungen der Medien aus der schulischen Ordnung reflexiv erschlossen.

\subsection{Bedeutung für die schulische Ordnung}

Abgucken \& Rechtschreibung: digitales Diktat Die Schüler(innen) schreiben auf den Tablets. Dabei kommt es immer wieder zu kleineren Entdeckungen (z.B. „Jetzt weiß ich wie man großschreibt! “). Auch die Rechtschreibprüfung der Software sorgt für Aufsehen und wird ergründet. Dieses Programmfeature moniert nicht nur ankreidend Fehler, sondern schlägt auch unumwunden und konstruktiv die richtige Schreibweise vor. Einige Kinder wirken fasziniert, andere ungläubig. „Hiermit mal ein Diktat schreiben!“ ruft ein Junge und ein anderer rollt mit den Augen: „Dann bekommt ja jeder eine Eins".

Auch in dieser Beobachtung werden die Medien vor dem Hintergrund des Schulischen eingeordnet. Die Merkmale und Kompetenzen der Software werden hier jedoch weniger aus dem Schulischen heraus erklärt, als vielmehr in ihrer Bedeutung für das Schulische reflektiert. Dies geschieht, indem über das Gedankenexperiment eines Diktats die Tragweite der entdeckten Softwarefunktionalität für die schulische Ordnung herausgestellt wird. Auch die darauffolgende (postwendende) Reaktion des Mitschülers nimmt Bezug auf die schulische Ordnung, die in dem Gedankenexperiment ad absurdum geführt werden würde: Mit einem solchen Tablet macht 
ein Diktat keinen Sinn, da es verhindern würde, dass sich die Schüler(innen) auf einer Notenskala verteilen. Es wird daher nicht zu diesem Szenario kommen. Mit Reflexionen wie diesen zeigen sich die Schul- und die mit ihnen verwickelten Medienkompetenzen der Kinder.

\section{Fazit: Kompetenzverwicklungen}

Ausgehend von der schulisch-konstituierten Andersartigkeit der digitalen Medien in der Unterrichtsinteraktion zeigte der Beitrag auf, wie Kinder - in ihrer Rolle als Schüler(innen) - das Grundschulische zugrunde legen. Sie machen das Schulische zu einer Heuristik für ihre Medienaneignung und -reflexion. Anders formuliert: Kinder in der Schule nähern sich den neuen Medien im tradierten Medium des Schulischen. Das schulische Kerngeschäft des Schreibens ist dabei beispielhaft als analytische Kategorie zentralisiert worden, zweierlei ließ sich systematisieren: Es wurde (1) sichtbar, wie Schüler(innen) aus der Schulordnung als Referenzsystem (Be-)Deutungen ableiten. Mit Blick auf die digitalen Medien wird das Schulische dabei für Befragungen, Einordnungen, Erklärungen und Sinnstiftungen herangezogen - es wird kompetent zur Vermittlung genutzt (,Damit keiner abschreibt, ne?“). Zudem zeigt sich (2), dass Kinder auch das Schulische vor dem Hintergrund der neuen Medien be- und hinterfragen bzw. Bedeutungen für die schulische Ordnung reflektieren („Dann bekommt ja jeder eine Eins“). Die Kompetenz der Kinder liegt nicht zuletzt darin begründet, dass sie die Funktionen der Geräte und die Funktionen des Schulischen aufeinander beziehen bzw. reflexiv miteinander verwickeln. Medien- und Schulkompetenzen zeigen sich als ineinander verschlungen und werden zu einer Amalgamierung. Die gezeigte Medienkompetenz in der Schulkultur scheint nicht das gleiche zu sein wie Medienkompetenz in der Familie (vgl. z. B. Chaudron 2015; Wiesemann et al. 2015) oder der Peer-Kultur (vgl. z. B. Ito 2010), eben weil sie in der Schule schulisch ist, weil andere situative Deutungsfolien und kulturelle Ressourcen vordergründig sind. Das (An-)Erkennen dieser Eigenständigkeit ist grundlegend für neue Perspektivierungen: Wenn sich Medien und Medienkompetenzen außerhalb der Schule anders konstituieren als innerhalb der Schule, verliert z. B. das Bild vom „digital divide“ (vgl. z. B. Buckingham 2007) seine Dramaturgie und wird zur Selbstverständlichkeit. Natürlich kann die digitale Kluft zwischen institutioneller und privater Nutzung weiterhin mit Blick auf infrastrukturelle Ausstattungen und Umgangsquantitäten problematisiert werden. Mit Blick auf ohnehin divergente Umgangsqualitäten erscheint es aber fraglich, ob diese Kluft eingeebnet werden kann. Für ein grundlegendes Verständnis von dem, was schulische Medienkompetenz in actu und in situ empirisch ist, gilt es die schulisch-medialen Bedeutungen, „Eigenartigkeiten“ und Verwicklungen weiter zu analysieren, die sich in den Praktiken der Akteure zeigen. Auf dieser Grundlage kann für die schulische Aufladung der Medien sensibilisiert werden, deren Vergegenwärtigung auch Professionalisierungspotentiale zu bieten vermag. Grundlegend für diese kann ein generiertes schulisches Verständnis von medialen Handlungsproblemen, Erschließungsstrategien und Involvierungsweisen im Alltag sein. Im Reflektieren dieser empirischen Schulspezifik von Medien(-kompetenz) liegen neue Relevanzen für Schul- und Theorieentwicklung. 
Funding Open Access funding provided by Projekt DEAL.

Open Access Dieser Artikel wird unter der Creative Commons Namensnennung 4.0 International Lizenz veröffentlicht, welche die Nutzung, Vervielfältigung, Bearbeitung, Verbreitung und Wiedergabe in jeglichem Medium und Format erlaubt, sofern Sie den/die ursprünglichen Autor(en) und die Quelle ordnungsgemäß nennen, einen Link zur Creative Commons Lizenz beifügen und angeben, ob Änderungen vorgenommen wurden.

Die in diesem Artikel enthaltenen Bilder und sonstiges Drittmaterial unterliegen ebenfalls der genannten Creative Commons Lizenz, sofern sich aus der Abbildungslegende nichts anderes ergibt. Sofern das betreffende Material nicht unter der genannten Creative Commons Lizenz steht und die betreffende Handlung nicht nach gesetzlichen Vorschriften erlaubt ist, ist für die oben aufgeführten Weiterverwendungen des Materials die Einwilligung des jeweiligen Rechteinhabers einzuholen.

Weitere Details zur Lizenz entnehmen Sie bitte der Lizenzinformation auf http://creativecommons.org/ licenses/by/4.0/deed.de.

\section{Literatur}

Ala-Mutka, K. (2011). Mapping digital competence. Towards a conceptual understanding. Luxembourg: Publications Office of the European Union.

Amann, K., \& Hirschauer, S. (1997). Die Befremdung der eigenen Kultur. Ein Programm. In S. Hirschauer \& K. Amann (Hrsg.), Die Befremdung der eigenen Kultur. Zur ethnographischen Herausforderung soziologischer Empirie (S. 7-52). Frankfurt a. M.: Suhrkamp.

Baacke, D. (Hrsg.). (1974). Kritische Medientheorien. Konzepte und Kommentare. Serie Kommunikation. München: Juventa.

Baacke, D. (1999). Medienkompetenz als zentrales Operationsfeld von Projekten. In D. Baacke, S. Kornblum, J. Lauffer, L. Mikos \& G. A. Thiele (Hrsg.), Handbuch Medien. Medienkompetenz: Modelle und Projekte (S. 31-35). Bonn: Bundeszentrale für Politische Bildung.

Beck, G., \& Scholz, G. (2000). Teilnehmende Beobachtung von Grundschulkindern. In F. Heinzel (Hrsg.), Methoden der Kindheitsforschung. Ein Überblick über Forschungszugänge zur kindlichen Perspektive (S. 147-170). Weinheim: Juventa.

Bettinger, P. (2018). Rekonstruktive Medienbildungsforschung. Die Analyse von Bildungsprozessen als Habitustransformationen in mediatisierten Lebenswelten. In T. Knaus (Hrsg.), Forschungswerkstatt Medienpädagogik 1. Aufl. Projekt - Theorie - Methode, (Bd. 2, S. 569-600). München: kopaed.

Bos, W., Eickelmann, B., Gerick, J., Goldhammer, F., Schaumburg, H., Schwippert, K., et al. (Hrsg.). (2014). ICILS 2013. Computer- und informationsbezogene Kompetenzen von Schülerinnen und Schülern in der 8. Jahrgangsstufe im internationalen Vergleich. Münster: Waxmann.

Buckingham, D. (2007). Media education goes digital: an introduction. Learning, Media and Technology, 32(2), 111-119.

Carretero, S., Vuorikari, R., \& Punie, Y. (2017). Digcomp 2.1. The digital competence framework for citizens. Luxembourg: Publications Office of the European Union.

Chaudron, S. (2015). Young children (0-8) and digital technology. A qualitative exploratory study across sevencountries. Luxembourg: Publications Office of the European Union.

Emerson, R. M., Fretz, R.I., \& Shaw, L.L. (1995). Writing ethnographic fieldnotes. Chicago: University of Chicago Press.

Ferrari, A. (2012). Digital competence in practice. An analysis of frameworks. Luxembourg: Publications Office of the European Union.

Geertz, C. (1983). Dichte Beschreibung. Frankfurt a. M.: Suhrkamp.

Heinzel, F. (2003). Methoden der Kindheitsforschung. Probleme und Lösungsansätze. In A. Prengel (Hrsg.), Im Interesse von Kindern? Forschungs- und Handlungsperspektiven in Pädagogik und Kinderpolitik (S. 123-135). Weinheim: Juventa.

Hengst, H. (2008). Kindheiten. In H. Willems (Hrsg.), Lehr(er)buch Soziologie. Für die pädagogischen und soziologischen Sudiengänge (1. Aufl. S. 551-581). Wiesbaden: Springer VS.

Honig, M.-S., Lange, A., \& Leu, H. R. (1999). Zur Einführung. In M.-S. Honig, A. Lange \& H. R. Leu (Hrsg.), Aus der Perspektive von Kindern? Zur Methodologie der Kindheitsforschung. Kindheiten, (Bd. 16, S. 9-32). Weinheim: Juventa-Verl.

Ito, M., et al. (2010). Hanging out, messing around and geeking out. Kids living and learning with new media. Cambridge: MIT Press. 
Kammerl, R. (2014). Enkulturationshilfen in der digitalen Gesellschaft. Diskurse als/oder Orientierung? In R. Kammerl, A. Unger, P. Grell \& T. Hug (Hrsg.), Diskursive und produktive Praktiken in der digitalen Kultur. Jahrbuch Medienpädagogik, (Bd. 11, S. 15-33). Wiesbaden: Springer VS.

Kelle, H., \& Breidenstein, G. (1996). Kinder als Akteure. Ethnographische Ansätze in der Kindheitsforschung. Zeitschrift für Sozialisationsforschung und Erziehungssoziologie, 16(1), 47-67.

KMK (2016). Bildung in der digitalen Welt. Strategie der Kultusministerkonferenz. Berlin: Sekretariat der Kultusministerkonferenz.

Krappmann, L., \& Oswald, H. (1995). Alltag der Schulkinder. Beobachtungen und Analysen von Interaktionen und Sozialbeziehungen. Kindheiten, Bd. 5. Weinheim: Juventa Verl.

Kübler, H.-D. (1996). Kompetenz der Kompetenz der Kompetenz.. Anmerkungen zur Lieblingsmetapher der Medienpaedagogik. Medien praktisch, 20(78), 11-15.

Leuthner, V. (2014). Kids unterwegs im WWW. „Digital Natives“ oder „Digitale Analphabeten“? Internetkompetenz bei Kindern und Jugendlichen zwischen Anspruch und Wirklichkeit - Eine Bestandsaufnahme. Tübingen: Universitätsbibliothek Tübingen.

Li, Y., \& Ranieri, M. (2010). Are 'digital natives' really digitally competent?-A study on Chinese teenagers. British Journal of Educational Technology, 41(6), 1029-1042.

Medienpädagogischer Forschungsverbund Südwest (Hrsg.). (2019). KIM-Studie 2018. Kindheit, Internet, Medien. Basisuntersuchung zum Medienumgang 6- bis 13-Jähriger. Stuttgart: Mpfs.

Moser, H. (2014). Die Krise der Repräsentation und ihre Folgen für die medienpädagogische Forschung. In A. Hartung-Griemberg, B. Schorb, H. Niesyto, H. Moser \& P. Grell (Hrsg.), Methodologie und Methoden medienpädagogischer Forschung. Jahrbuch Medienpädagogik, (Bd. 10, S. 55-73). Wiesbaden: Springer VS.

Muchow, M., \& Muchow, H.H. (1935). Der Lebensraum des Großstadtkindes. Der Ertrag der Hamburger Erziehungsbewegung, Bd. 2. Hamburg: Riegel.

Münker, S. (2013). Media in use: how the practice shapes the mediality of media. Distinktion: Journal of Social Theory, 14(3), 246-253.

Münte-Goussar, S., \& Grünberger, N. (2019). Medienbildung und die Kultur der Schule. Praxistheoretische Zugänge zur Erforschung von Schule in einer mediatisierten Gesellschaft. In T. Knaus (Hrsg.), Forschungswerkstatt Medienpädagogik. Projekt - Theorie - Methode, (Bd. 3, S. 911-942). München: kopaed. https://publ.forschungswerkstatt-medienpaedagogik.de/download/FWMP-Band-3_MuenteGoussar-Gr\%C3\%BCnberger_19Jun13.pdf.

Prensky, M. (2001). Digital natives, digital immigrants. On The Horizon, 9(5), 1-6. http://www. marcprensky.com/writing/Prensky\%20-\%20Digital\%20Natives, \%20Digital\%20Immigrants\%20$\%$ 20Part1.pdf.

Rode, D., \& Stern, M. (2017). Praktiken der Objektivierung und Subjektivierung. Eine praxeologische Perspektive auf Medienkompetenzerwerb im Tanzunterricht. In K. Mayrberger, J. Fromme, P. Grell \& T. Hug (Hrsg.), Vernetzt und entgrenzt. Gestaltung von Lernumgebungen mit digitalen Medien. Jahrbuch Medienpädagogik, (Bd. 13, S. 231-243). Wiesbaden: Springer VS.

Schorb, B., \& Theunert, H. (2000). Kontextuelles Verstehen der Medienaneignung. In I. Paus-Hasebrink \& B. Schorb (Hrsg.), Qualitative Kinder- und Jugendmedienforschung. Theorie und Methoden ; ein Arbeitsbuch. München: KoPäd-Verl.

Schulmeister, R. (2012). Vom Mythos der Digital Natives und der Net Generation. Berufsbildung in Wissenschaft und Praxis. Zeitschrift des Bundesinstituts für Berufsbildung, 41(3), 42-46.

Schulz, M. (2014). Ethnografische Beobachtung. In A. Tillmann, S. Fleischer \& K.-U. Hugger (Hrsg.), Handbuch Kinder und Medien. Digitale Kultur und Kommunikation, (Bd. 1, S. 225-235). Wiesbaden: Springer VS.

Strauss, A. L. (1994). Grundlagen qualitativer Sozialforschung. Datenanalyse und Theoriebildung in der empirischen soziologischen Forschung. München: Fink.

Sutter, T. (2014). Selbstsozialisation und Medienbildung. In W. Marotzki \& N. Meder (Hrsg.), Perspektiven der Medienbildung. Medienbildung und Gesellschaft, (Bd. 27, S. 71-90). Wiesbaden: Springer VS.

Treumann, K. P., Meister, D. M., Sander, U., Burkatzki, E., Hagedorn, J., Kämmerer, M., Strotmann, M., \& Wegener, C. (2007). Medienhandeln Jugendlicher. Mediennutzung und Medienkompetenz. Bielefelder Medienkompetenzmodell (1. Aufl.). Wiesbaden: Springer VS.

Tulodziecki, G. (1997). Medien in Erziehung und Bildung. Grundlagen und Beispiele einer handlungsund entwicklungsorientierten Medienpädagogik (3. Aufl.). Bad Heilbrunn: Klinkhardt.

Tulodziecki, G., Herzig, B., \& Aßmann, S. (2018). Der Diskurs um Medienkompetenz und Medienbildung und seine Bedeutung für die Theorieentwicklung in der Medienpädagogik. In I. M. Lüders \& W. Me- 
seth (Hrsg.), Theorieentwicklungen in der Erziehungswissenschaft. Befunde - Problemanzeigen Desiderata. Klinkhardt forschung, (Bd. 44, S. 37-50). Bad Heilbrunn: Klinkhardt.

Wiesemann, J., Eisenmann, C., \& Fürtig, I. (2015). Medienpraxis in der (frühen) Kindheit. Ethnografische Exploration des familiären Smartphonegebrauchs. merzWissenschaft, 59(6), 39-47.

Zinnecker, J. (2001). Stadtkids. Kinderleben zwischen Straße und Schule. Kindheiten, Bd. 20. Weinheim: Juventa-Verl. 